\title{
Are Universities Ready to Recognize Open Online Learning?
}

\author{
Margarita Tereseviciene ${ }^{1}$, Elena Trepule ${ }^{1}$, Estela Dauksiene ${ }^{1}$, Giedre Tamoliune ${ }^{1} \&{\text { Nilza } \text { Costa }^{2}}^{2}$ \\ ${ }^{1}$ Education Academy, Vytautas Magnus University, Kaunas, Lithuania \\ ${ }^{2}$ Research Centre for Didactics and Technology in Teacher Education, University of Aveiro, Aveiro, Portugal \\ Correspondence: Margarita Tereseviciene, Education Academy, Vytautas Magnus University, Kaunas, Lithuania. \\ E-mail: margarita.tereseviciene@vdu.lt
}

Received: September 13, 2019

doi:10.5539/ies.v13n2p21
Accepted: October 19, 2019 Online Published: January 29, 2020

URL: https://doi.org/10.5539/ies.v13n2p21

\begin{abstract}
Fast development of technologies, changing needs of digital learners and other aspects of the digital era have had a major impact on universities and their learning management procedures. Access to information online, possibilities of open online learning and need to manage one's time lead to the changed profile of today's students and their need to recognize their prior knowledge or skills. This brings a challenge for universities to adapt their procedures of prior learning recognition. This research aims at identifying requirements for universities to recognize open online learning $(\mathrm{OOL})$, focusing on the qualitative analysis of insights and experiences of experts who are knowledgeable and experienced in the field of OOL. Although OOL recognition procedures tend to be similar as in the recognition of other types of learning, the universities face external challenges, coming from labour market, as well as reserved, if not negative, attitudes towards openness and lack of trust in OOL by traditional universities, thus distinguishing the OOL recognition process as being far from accepted practices. The research findings highlight several prospective requirements for universities set to recognize OOL.
\end{abstract}

Keywords: open online learning (OOL), recognition, requirements for recognition, universities

\section{Introduction and State of the Art Research}

With the growth of open online learning (OOL) offers, learners are taking increasing advantage of getting their non-formal education and continuous professional development (CPD) by way of OOL. Paradoxically, learners happen to gain academic knowledge by attending a variety of different courses that, in some cases, offer state of the art and edgy knowledge which is not offered by universities. Besides, the achievements of those courses are willingly accepted and recognized by employers. The employers increasingly tend to make no difference in recognizing the OOL or other forms of learning. A survey of 103 employers in the US revealed that $83 \%$ considered MOOCs to be a suitable source of education for CPD (Radford et al., 2014). When learners enrol to university degree studies and expect to save time and a certain fee exemption, they naturally seek university recognition of their learning experience or skills acquired in OOL courses or activities. Meanwhile universities lack procedures, or trust, and face emerging challenges for the recognition of the knowledge or skills acquired by various forms open learning.

Therefore, this research makes an attempt to outline requirements for universities in the recognition of OOL by interviewing experts in education on how selected universities at international level have been a) valuing OOL, b) recognizing OOL and c) what suggestions emerge to improve those two dimensions. Thus the research question what are the requirements for universities to recognize open online learning $(O O L)$ ? - implies understanding the practices of selected universities at the international level, of how they value and recognize OOL and what recommendations can be drawn for the benefit of potential academic members of the recognition of OOL.

Open online learning (OOL) is defined by the typical features of open distance learning (Cole, Shelley, \& Swartz, 2014; Simpson, 2013; Thorpe, 2002; Tait, 2000):

- access to open or distance courses;

- open and flexible learning online;

- collaboration of open online learning activity learners in terms of support and course content, as well as

- development of new open knowledge and 
- $\quad$ sharing learning outcomes among learners, with teachers, and wider community.

Witthaus et al. (2016) refer to three types of open learning in the field of online and distance learning with different levels of openness and different possibilities of learning assessment and recognition, and illustrate their levels of recognition (based on Langen \& Bosch, 2013) as: (i) open education and open universities, (ii) massive open online courses (MOOCs), (iii) open educational resources (OER).

Assessment and recognition are the major concepts referred in the report on validation of non-formal MOOC-based learning (Witthaus et al., 2016); they are mostly related to higher education and Bologna process. Assessment in this context may have two meanings: how assessment was performed in an OOL (i.e. assessment of learning outcomes of the OOL course or other type of activity) and assessment of the credentials provided by the OOL provider. In this research we focus more on the assessment of the credentials for the recognition of the learners' learning achievements.

Recognition, according to UNESCO (2012, p. 11), is defined as a process of granting official status to learning outcomes and/or competences, which can lead to the acknowledgement of their value in society. Recognition of learning in other contexts, including open and online learning, could be performed by the universities or by the employers who are hiring the learners. The employers are increasingly more interested in the skills and performance rather than the nature of their acquisition, while universities are restrained by the formal requirements for recognition and quality expectations (Harris \& Wihak, 2018).

In higher education the major goals of recognition of open non-formal learning (Witthaus et al., 2016) are related to (i) entry to programmes offered by higher education institutions; (ii) exemption from part of the programme or (iii) awarding a full higher education qualification. Open non-formal learning could be defined as a conscious form of learning when distance learning is used.

The existing good practices of assessment and recognition of OOL are still rather scarce and unsystematic, not qualifying as any consistent practices or systems that could already be functioning in universities. If the universities rely on the assessment procedures performed by different course providers, those, in their turn, should provide sufficiently detailed information on the assessed course in terms of the course volume in hours, credits, learning outcomes, levels of achievement, ways of assessment, among others. More than that, it is crucial to establish a connection between the assessing institution that provides the courses and assesses learner achievements and the recognizing institution that recognizes the learner's course result assessment towards a part of a degree to allow coherent processes of the recognition of OOL.

From a political point of view provisions for universities regarding the assessment and recognition of open online learning have been highlighted. However, these policies are only emerging, and our team failed to find explicit and consistent cases of such policy in any particular country or particular university. The existing policies found are still mostly on very strategically political international levels of transnational organizations, such as UNESCO or the EU. The World OER Congress held at UNESCO in 2012 encouraged the development of mechanisms for the assessment and certification of learning outcomes achieved through OER. An EU Council recommendation (2012) committed the Member states to introduce systems for validation of non-formal and informal learning, also including learning through OER. A CEDEFOP report on validation of OER (2016) mentioned lack of data about validation of learning acquired through OER and suggested fivefold recommendations: (i) share knowledge and spread good practice on the validation of OER-derived learning across the formal education sector, (ii) expand the options of what can be validated, to include full qualifications, (iii) develop and make stakeholders aware of the options for validation of learning outcomes from participation in OER/MOOCs and the different benefits of these options, in particular in different European education and labour market contexts, (iv) improve measures to link learning derived from the use of OER with other generic systems for the validation of non-formal and informal learning, (v) invest in high-quality assessment systems.

A study of MOOC offerings highlighted six elements that are central for the future recognition by other higher education institutions or employers: (i) identity verification of the learner, (ii) suitable supervised assessment, (iii) informative credentials, such as (digital) certificates or, in the near future, online badges that acknowledge learning, (iv) quality assurance, (v) award of credit points, (vi) partnerships and collaboration with potentially recognizing institutions or bodies (Witthaus et al., 2016, p. 69).

Camillieri and Tannhäuser (2012), Conrad and McGreal (2012), Harris and Wihak (2018), Friesen and Wihak (2013), and Mackintosh et al. (2011) investigate the possibilities of using the systems of recognition of prior learning (RPL) for the recognition of OOL. In some cases, there are references to Recognition of Non-formal Education (RNFE) or Prior Learning Assessment and Recognition (PLAR), and in all these cases the idea is that traditional prior learning recognition procedures might be used for OOL. 
An already quite widely established practice of Assessment and Recognition of Prior Learning (RPL) could also be used to recognize OER learning (Camillieri \& Tannhäuser, 2012). Following the EU 2012 recommendation to introduce systems for validation of nonformal and informal learning, including through OER, learners could get OER learning validated and obtain partly or full qualifications based on the validated experiences. The UNESCO Paris Declaration of 2012 also emphasizes the need to encourage the development of mechanisms for the assessment and certification of learning outcomes achieved through OERs.

The research carried out by institutions from 13 countries of the world led by the Open Educational Resource University (OERu) in New Zealand found out that existing RPL practices are usually very much dependent on the individual institutional policies and practices (Conrad \& McGreal, 2012). Of the 31 institutions that participated in the research on recognition of learning through OER only 22 practiced RPL (71\%) while other 17 (55\%) allowed the transfer of credit, and institutions used a variety of different practices for that.

Friesen and Wihak (2013) investigated how learners could get learning from OER and MOOCs assessed and accredited through the system of (PLAR. They concluded that despite the existing pathways of using course-based portfolios or college exam banks, credentialing was still ad hoc and not systemic, though becoming more widespread and recognised in PLAR and OER communities. Mackintosh et al. (2011) notice that PLAR is already a recognised process to evaluate and recognize learning outside formal institutions for independent learning and could be rather well applied for assessment of open learning. Nevertheless PLAR methodologies are very much time and human resource consuming, alongside with the fact that in different countries methodologies vary. Besides, in case of MOOCs, the big numbers of learners make assessment problematic in terms of staffing (Chauhan, 2014) and often result in using automated machine assessment procedures.

From the above, our study attempts to contribute to the development of knowledge in the domain of the recognition and assessment o OOL and so to bring contributions needed in such domain.

\section{Method}

\subsection{Research Context}

The current study is part of a large research project on the needs of digital networked society for open and online learning. As it was discussed above, recognition is an important criterion for society members interested and participating in OOL. The initial analysis of the research topic disclosed the urgent need for discussing the process of recognition more deeply. Being a complex process it confirms that diverse approaches should be considered when discussing the existing practices and challenges. Therefore, this study is focusing on the insights and experiences of experts who are knowledgeable and experienced in the field of OOL and/or recognition in higher education or at policy level.

The method-qualitative research inquiry-was chosen to disclose the research participants' approaches to and experiences with the requirements for universities to recognize open online learning.

\subsection{Sampling and Participant Characteristics}

The qualitative research inquiry used purposive sampling that allows identifying and selecting people who are most knowledgeable in the research area, share similar characteristics, and are able to provide data related to the research question (Creswell, 2013). Two criteria applied for research participants: they had to be 1) experts in OOL and/or recognition of open learning in higher education; 2) representatives of higher education institutions or higher education policy advisers. Twelve international experts having knowledge of the recognition of open online learning agreed to share their experiences and insights on the research topic: eight research participants represented researchers-practitioners of OOL at higher education institutions, and the other four were policy advisers for OOL. Interviews with the experts allowed combining approaches of the respondents representing policy making and research fields and allowed to disclose the complexity of the research problem.

Research participants represented public and private institutions from eight European countries. The aim of the research was not to compare data different institutions, but rather to focus on general requirements for recognition. The international group of research participants allowed ensuring that existing requirements, challenges and practices were not related to specific national or institutional culture; instead, it demonstrated an international and rather wholistic approach towards the research problem.

\subsection{Fact and Attitude Collection and Management}

Semi-structured interviews were conducted to collect the most relevant insights and opinions relating to the research question. The interviews were pooled for data analysis in January and February 2019. Interview questions were non-directive and open-ended, based on the topics, constructed on the basis of the theoretical analysis. This 
method helps researchers to follow the set of questions prepared in advance, develop them or reorganise the sequence of questions when needed. In addition (Kvale \& Brinkmann, 2009), it allows the interviewees to express their ideas using the concepts and terms that are natural for their thinking and talking, rather than pertain to something constructed artificially.

At the initial stage, experts were contacted personally by researchers, introduced to the research aim and interview guidelines, and asked for their consent to participate in the research. Each research participant agreed to share his or her expertise via face-to-face or synchronous online meetings, using videoconferencing tools. The research participants were invited to share their expertise and knowledge on the existing practices of recognition process of open online learning, on the challenges and requirements for this process to be successful, and on the ideas of how universities should prepare for digital credentialization. Interviews were audiotaped and transcribed right afterwards. All interviews were collected in English, to ensure the conformity of the ideas expressed and concepts used.

Research participants were given the codes (I.1, I.2, I.3...I.12) to ensure confidentiality of research results, and only the group of leading researchers had access to the real names and surnames of the interviewees.

\subsection{Data Analysis}

Qualitative content analysis was used to analyse research data. It encompasses a set of methods that help to "explore explicit or covert meaning in text" (Bernard \& Ryan, 2010, p. 287) and identify " $<\ldots>$ categories that grasp favourable or unfavourable attitudes or representations of these" (Jupp, 2006, p. 40). Even though the content analysis is referred to be deductive rather than inductive in its nature, and requires to count the "number of 'mentions' within each category" (Silverman, 2007, p. 163), when applied in qualitative analysis of texts, it aims to "understand the participants' categories" (Silverman, 2007, pp. 163-164). The qualitative content analysis is closely related to the research context, where the researched processes appear. It allows describing the researched phenomenon based on unique experiences of the research participants.

Inductive qualitative content analysis was conducted following the main steps: 1) formulating the research question; 2) selecting sets of texts responding to the research question; 3) open coding; 4) constructing manifesting categories and subcategories; 5) choosing the most relevant extracts of the texts to illustrate specific sub-categories (Creswell, 2013; Bernard \& Ryan, 2010). To ensure the validity of research results, the authors of this paper reviewed categories and subcategories, and discussed the compliance among the manifesting categories, subcategories and excerpts of interviews.

Following this procedure of data analysis, three categories were constructed as disclosing research problem, namely, external trends or challenges, institutional readiness, and data from OOL providers. Each category comprised sub-themes, disclosing different aspects of each category and helping to better understand the requirements set for universities to recognize open online learning.

\subsection{Research Limitations}

Interviews with policy advisers for OOL and researchers at higher education institutions allowed disclosing the research problem from two perspectives, while there are more stakeholders who could contribute to this discussion and disclose new perspectives about OOL recognition, such as students or representatives of labour market. It is assumed that these research limitations may develop into a potential future research in this field.

\section{Results}

The research findings identified several challenging factors which are related to and lead to the requirements for universities for OOL recognition. Those challenging factors are related to i) external trends; ii) internal readiness of university; and iii) learner provided evidence on open online learning.

\subsection{External Trends or Challenges}

The experts identified different external factors and obstacles in relation to OOL recognition: those which come from OOL variety, too many standards, guidance and regulations, different attitudes and trust in open learning, fast development and flexibility of labour market, and changing learners and their needs. Those factors require fundamental and strategic changes in the universities, and eventually lead to change in internal university practices.

\subsubsection{Following the Same Procedures as in Traditional Recognition}

In general, the experts indicated no difference in recognizing course learning outcomes or skills in traditional and open online learning. However, they emphasized the existence of a variety of recognition regulating documents, guidelines and standards at national levels and within Europe which tend to ensure the quality of learning 
processes. References to existing common documentation models, like the level of qualification in the European Qualification framework (EQF) or National Qualification framework (NQF), were pointed out as to be followed and applied in open and online learning.

"...if you look at it purely from conceptual standpoint, the model of learning should make absolutely no differences. Whatever is used in traditional learning can also be used online." (I.11).

The challenging factors here are the non-existent framework of OOL which leads to a variety of learning forms and modes.

“...we have a very wide field of open and online learning opportunities, but we have no framework that frames it sufficiently" (I.4).

It is not recommended to unify these forms of OOL, as they should be appreciated, but to be properly recognized they need to have certain features described or "translated" to suit universities' procedures. Also, it is important not to set too high standards for open online learning-the same as in traditional recognitions should prevail:

"I believe we shouldn't set the standards too high, because we tend to have higher standards when we talk about online learning than we have when we talk about normal traditional, higher education. So, I think we have to be flexible on that, like making sure, for example, when I have an online course, and I have an assessment type as ... an essay to hand in, when I have an essay within a traditional education course, I don't ask them to send a video of them writing the essay, and it happens that a student betrays and not he or she writes the essay, but someone else, and I think that level of trust, we should also have in open and online settings." (I.4)

Nevertheless, universities trust the credentials provided within the traditional process of recognition, while for recognizing the OOL, more evidence may be provided, but an issue of trust is still open:

"....if we compare this recognition with the traditional forms ... some details and elements are not visible. ... we can track much more details and objective data in virtual space." (I.1)

\subsubsection{Openness and Trust in Open Online Learning}

Universities need to open up for a number of reasons. The experts indicated a few: first, to stay competitive in providing the new and fast changing knowledge while collaborating with other universities. As one of the experts pointed out:

"....if my engineering programme is not focused ... on automotive, but a partner university or any competent university in Spain does, ... there I can study Artificial Intelligence in the area of automotive, I should open up this line for the students, because if he will not do it there, he will do it in a private market, that's where he will get it, so they [universities] have to open up."(I.9)

Transparency was indicated as another reason for universities to become or stay open. Experts consider open and online learning, even if it is not really open in all aspects, as much more transparent and more objective, because we can "... find and track everything in digital format" (I.1). Transparency is not only related to tracking students' work and activities, but also to recognition procedures. At this point experts see the level of trust in OOL to be the main obstacle for OOL assessment and recognition to become a widely accepted practice.

"trust is a core issue, and this trust has not been reached, the trust level that you need in order to have assessment and recognition practices that are widely accepted" (I.4)

University openness also means the change in teacher attitude towards openness; sharing and use of online learning content created by others; the new modes of teaching and learning, which also lead to changes in institutional values and culture. The experts indicated that there is a lot of resistance, or lack of trust in OOL recognition, especially among those who are not practicing open or online learning.

"those who teach online, and the majority of them come from open universities, but also from blended universities... for them, assessment and recognition is already accepted practice. For those who do not have experience and who do not have regulations for example on how to implement these procedures, it is not accepted practice". (I.1)

It depends a lot on the university practice, but

"if the student can't reasonably prove that they've done some kind of open learning, open activities and they're roughly compatible then they're happy to get people onto the courses, but that wouldn't mean that they'd let them use it to substitute part of the learning that's actually coming online" (I.5)

However, some universities try new solutions that may work not perfectly, they try them to see advantages and disadvantages, and if such universities come with the good solutions, they would be fostering idea of recognition 
forward.

\subsection{Institutional Readiness}

The process of assessment and recognition is important for both-for those who wish to recognize their achievements, and those to recognize them. To prepare for recognition of OOL inside the university and set internal transparent procedures is the most important thing, so that the learners would be "confident on how and when, and through which type of learning they can reach recognition" (I.1).

\subsubsection{Preparedness of Staff and Programmes}

Recognition of OOL is usually a new practice for university staff and therefore it might be met by some resistance to be implemented. Also, lack of skills and experience as well as non-existent procedures on how to implement the OOL recognition are hindering this from becoming an accepted practice.

Nevertheless, from university point of view, the recognition process limits the number of courses a student has to take in the programme, which means that the student has to take a smaller number of courses, or teachers have less students in the course:

"if you think about it, what the university business model is? To get students in the students' register for the courses, then they pay for the courses, and they start to learn. So, in a way it's like a kind of mechanism for attracting students from other kinds of backgrounds, because at the end of the day you want them paying to do your courses." (I.5)

In such a way the recognition not only challenges the university business model, but the challenge is getting greater as more stakeholders are involved. Therefore, not only professionals in recognition, human resource managers, but also academic staff need to be trained to be aware of procedures of OOL recognition and possible activities to reach it. Besides, more collaboration between stakeholders and efficient coordination of the process is implied.

Experts suggest the course and programme designers to:

"think how to divide curriculum on the basis of learning results to such small units that would be logical for teachers and study programme committees, but at the same time that would be able to describe in terms of skills and competencies, there's specific ones, so that they can be prepared for micro credentialization of curriculum, of courses." (I.1)

It is quite clear that the course granularity or modularity is to be focused on and applied by the course or programme designers to better correspond the learners' needs.

\subsubsection{Readiness for Digital Format and Tools}

Universities are getting modernized, digitalized and technology equipped, they prefer not only to test innovations, but also stay innovative, and use technologies in their management processes. For the universities to be ready for assessment and recognition of digital evidence and credentials, digital format evidence and credentials should be ready for acceptance. This also means that universities have to have digital information systems and follow the same standards as other institutions or open learning providers do. Also, universities should

"establish the system itself, which would be synchronized with the existing, or planned to be designed in the future, digital credentialization platforms, so that university is prepared to issue diploma, also in digital formats, which can be divided into smaller units on the basis, maybe, of academic certificates, or even certificates for certain competencies and skills to be issued to learners, because they would not need paper, they would just need some confirmation that the learner is able to do certain things, and in terms of skills. So, these digital certificates, small certificates should be prepared in such technical format that they can be easily integrated into other platforms." (I.1)

There is also a need of a student information system in order to create and promote "culture and attitudes of data collection" (I.11), which allows to collect, document and share all the information about the courses, about student experiences, and results.

The experts also mentioned privileges in the assessment and recognition of OOL as everything should not be paper based anymore, and universities can follow up quite easily. The assessment of OOL can include some innovative tools, e.g., videos, multimedia and simulations, and other forms, such as self and collaborative peer assessment. And if we compare this recognition with the traditional forms:

"...we of course can have a lot of things in common, but in that case, people should print it out and have it in physical, tangible format and bring them." (I.12).

We can see this is different from recognizing the OOL, as there the whole format is digital, it uses innovative tools, 
and exploits all credentials possibilities.

For those who develop OOL courses, it is advisable not just to use different learning environments, but also organize more frequent assessments:

"In this open online environment, we do frequent evaluation, many times, it is not compulsory, but it is helpful for the interactivity with the material" (I.2)

Thus, introduction of digital credentialization, preparation of documentation on digital badges, digital information systems to accept digital evidence, and recognition procedures could be the first steps to start, as suggested by the research experts.

\subsubsection{Quality Assurance and Standardization}

There is a wide field of open and online learning opportunities, thus questions on the quality of the course and the quality of credentials issued usually come up. Yet, there is no framework that frames it sufficiently when recognition is in progress. To ensure quality, experts suggest setting standards, and the reason for that is:

"...because it is not clear what open online course is, and what the standards are that are defined for such a course. And because it leads to a lot of insecurity amongst credential evaluators within higher education institution, but also amongst students, who would like to know if they get their learning recognized before they actually study" (I.4)

It also should be stressed that the same existing standards that are valid in formal learning should be applied in open learning: in

"the formal recognition, there are standards, these standards have been set, and these standards should be applied, ... And these standards, they are in my opinion exactly the same, no matter if it's prior offline learning, ... or if someone did a formal online learning." (I.4)

The second observation that comes up very often is the quality of the assessment: any recognition needs to consider documenting how the assessment is done. The quality of assessment is more discussed in the following (3.3.1) section, here it needs to be noted that

"Recognition ... should be based on the quality of your course design and on your assessment procedures, ... any recognition framework needs to take into account documenting how you have actually verified the identity and how you've done the assessment" (I.11).

The experts discuss the possibility of highlighting similarities and differences of open online courses and comparing them with traditionally defined standards of the European higher education area, based on ECTS guidelines. They propose some kind of "translation" process:

"...and if you do such a translation process, only then it becomes possible to understand for a credential evaluator from the university to understand what this open online learning actually means..." (I.4)

The shared insecurity and lack of good practice examples, when standards higher than those at universities tend to be set for online learning, were stressed by the experts. This can be interpreted in such a way that well defined standards could increase trust among higher education institutions.

\subsubsection{Networking with Stakeholders}

Universities are getting involved in networks more and more, due to the assessment and recognition issues. In the digitalized century and in the networked society, universities cannot survive if they perceive themselves as a lone actor:

"They can only survive in strong networks and that includes the question of credentialization, openness, transparency, all these aspects" (I.11),

Universities tend to setup networks with those who could participate in e-assessments based on an open online learning opportunity. Universities are getting more successful with this; they are improving their practices and methods of assessment and recognition:

"For example, Coursera now provides a full Master's degree with some American university... They do it in collaboration with that university, so what that means is that university recognizes that Coursera is a good enough resource for them to grant a Master's degree to someone. So, the practices are always improving". (I.7).

Therefore, the future prospects of universities are with strong networks, and that, in its turn, includes the questions of openness, transparency, trust, and credentialization. 


\subsection{Requirements Set for OOL Providers}

Traditional recognition is when a higher education institution accepts the degree or credential of another institution and recognizes it either because they know the institution, its reputation and ranking and trust the ECTS credits or because they have cooperation with them. In such cases they would find it relatively easy to recognize it without even considering how learning and assessment was organized, how learner identity was validated or, in the worst case, whether the learner cheated getting the credential.

\subsubsection{Data about OOL Provider}

When it comes to recognition in OOL, the concern is who the provider of OOL is: whether it is provided by another, possibly well-known university, or it is provided by a company, which is not known at all. In such cases different recognition processes would be applied.

"The problem with an open and online learning is that it is sometimes not clear if, for example, a massive open online course is actually provided by a higher education institution or not. And that leads to different recognition processes that are possible to follow" (I.4).

It implies that trust in the issuer is an important factor when recognizing OOL, therefore networking could be helpful to promote or simplify recognition procedures.

\subsubsection{Data about Assessment Quality}

The quality of assessment in OOL is relevant and directly related to the trust issue, as it deals with assessment in a purely online or remote environment. It is quite agreed that many of the elements of assessment are very similar to the traditional way of learning: those would be assignments, papers, checking, lab work reports, the only difference being that those are digitally submitted. In the case of OOL, these have greater value as evidence, because they can be stored and shared more easily than papers that are filed in a folder. And very often the assessment could happen overtime in a monitored way, so even the trace of assessment could be better followed in online settings. Yet somehow, because of the trust issues in online learning and online learner identity, and time consumption in doing the assessment, there exists a big gap between the traditional and open online assessment. And although the elements are the same, there is more scepticism towards the open and online assessment.

In expert observations, different assessment quality assurance scenarios are available. First of all, the assessment could be made in offline places, as reflected by an expert:

"Regarding the assessment, I'd say it's the same, we have open online learning opportunities that provide e-assessments in offline places, that is what mostly works throughout Europe a lot, and has been piloted in that field, and then assessment is basically the same"(I.4).

Secondly, when assessment is provided online, the requirement for the process of OOL assessment should include learner identification, learner authentication, process description, and technical requirements: how participants should be connected; a description of the conditions in remote locations to secure confidence that this is the right person in the right place and doing the right thing. All of those factors should provide for validity and recognition.

"So, learner identification, learner authentication and then the process should be described in the way that it leaves no room for hesitation for the teacher and for the organization behind the teacher, that everything is crystal clear and transparent. For example, using web cameras or even audio and video collections, or let's say keystrokes identification, so if I use the same keyword, the computer may recognize that it is the same person as well, then we can recognize people from camera, face recognition, algorithm and other things" (I.1)

Obviously, a variety of techniques should be employed to ensure learner identification, authentication and quality of online learning and assessment, such as: biometrics (facial recognition, voice recognition, keystroke dynamics), document analysis, plagiarism tools, security techniques (time stamp, digital signature), etc.

\subsubsection{Data about Credentials and Their Metadata}

Recognition in open and online learning is ensured through evaluation of digital credentials, such as (open) digital badges, digital certificates and other micro-credentials. This suggests opportunities for greater granularity of learning and recognition. Evidence of what is done and achieved can be collected by using technologies. In many situations this allows to get more objective data if compared with traditional recognition. Although technology allows for more data, OOL recognition is most probably an objective of the future. Experts share that there is no one broadly accepted practice of online learning recognition at institutional level. Of course, experience of many universities which currently are most advanced in the field (such as universities from the USA, UK, Germany, France, Cyprus, Finland, etc.) has been mentioned, however, recognition could lead to different levels of formalization of credentials-usually an exemption from an entrance exam or an award of ECTS credits in major or 
minor programmes.

“... a few credits can be collected if open learning courses are taken and made with an assessment, but normally those are just kind for an extra or free learning subjects, which are freely chosen, those kind of things, but normally the recognition, if it is not strictly in the programme of the university, is not seen as something valuable, it is just of a free time learning" (I.3)

The growth in different types of credentials-from increasingly popular associate degrees to modern IT certifications-has been happening progressively over the last several years. It becomes the learners' currency for accessing opportunities as a function of the educational investment that they have made. If they receive a well-known diploma that is recognizable by an employer, it is perfect for the learner, but even this has to be sophisticated, standardized, recognizable, and machine readable by the employer systems. Like in one of the examples, provided by our experts:

"over ninety percent of fortune five hundred companies use keyword search of CVs which are submitted to them, so if those're dispatches and digital credentials, they probably will be accepted or at least they will be eligible for the keyword search; if that's just a bunch of upload these pdfs or even worse paper documents, you're not even going to get through the door, so that's where I think the digital part comes in, it allows for this ideal efficiency of let's say having a lot of information about having automatic ways to synthesize it and the way the employer can still look at. "(I.11)

The metadata displayed by credentials can also provide a basis for the recognition of OOL at university. Any credential, whether a certificate or an open badge, needs to provide information on the provider, the participant, the course workload, level of learning or certificate provided, the overall result, assessment type and the grade. However, experts notice the need to change the culture of institutions towards the data collection:

"so if you really want to get into micro-credentialization, I think it's really about saying okay we're going to create a structure at the institution where we can document all the information about the courses, all the information about the student experiences, all the evaluation and bring them together in one platform" (I.2).

The credential metadata initiative is driven by the idea to store the learning achievements one has collected over time and the underlying meta-data thus making it transparent and machine readable. The information contained in the digital credential provides grounds for future decisions on recognition, so it should be as much detailed as possible.

To sum it up, the research findings reveal external challenging factors in the process of OOL recognition. These factors are related to external forces and decisions, and the internal requirements for universities or OOL providers. Those include internal university readiness to take decisions on OOL recognition and information requirements from providers (see Figure 1).

As experts indicated, OOL recognition closely relates to political decisions, such as standards and guidelines that lead recognition procedures within Europe, that those should be the same, when it comes to OOL. However, OOL recognition means changes in institutional values and culture, a change of attitude towards openness, new procedures, and technological readiness for digital credentialization. The phenomenon of OOL has lack of trust in the society, and the trust is the main obstacle to have successful recognition practices that in traditional learning are widely accepted.

On the other hand, enterprises tend to recognize the competencies their employees demonstrate and are more flexible than universities. The universities are too slow to adapt to these changes in the market, while the students are adapting faster to the needs of industry. In relation to that OOL recognition for universities means new structural changes, testing internal documents, information gathering on student experience and evaluation on one university platform, as well as curriculum reconstruction in terms of skills and competencies. 


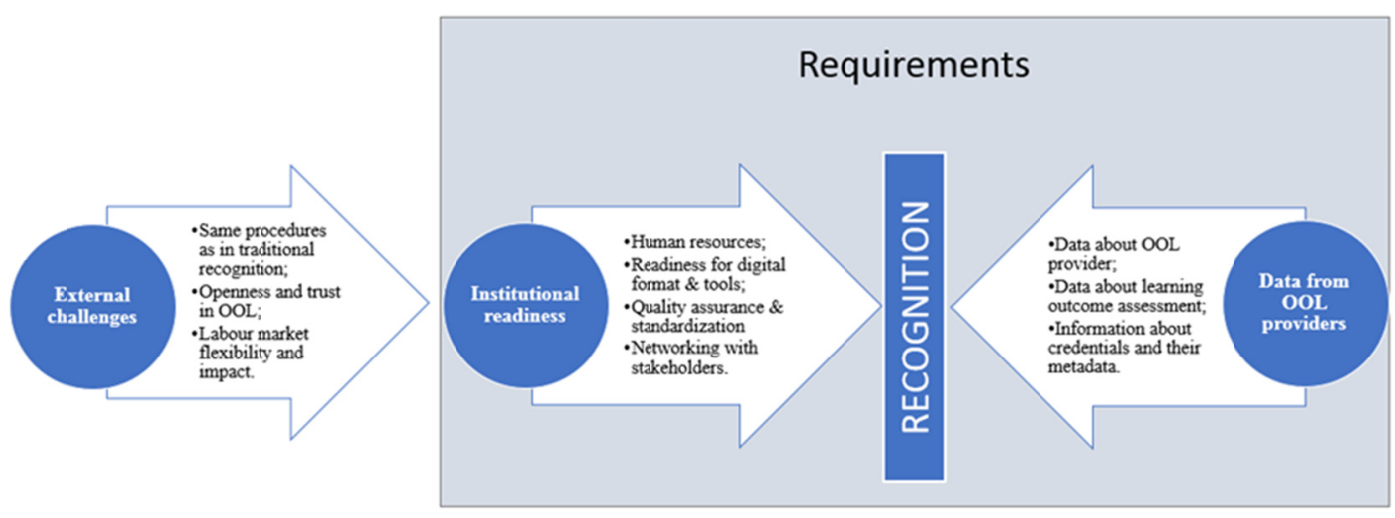

Figure 1. Requirements for universities to recognize open online learning

Seeking to prepare for the recognition of OOL, first the university needs strategic decisions and setting internal transparent procedures, in order that the staff and learners are confident on how and when, and through which type of learning OOL could be recognized. This also means that professionals in recognition, human resource managers, as well as academic staff should be instructed on procedures of recognition and possible activities around it. In such a way clearly defined quality assurance procedures and standards could increase trust among all stakeholders. The role of stakeholders, when OOL recognition is in focus, is of high importance, as strong networks with a variety of stakeholders contribute to easier solutions when it comes to questions of openness, transparency, trust, and digital credentialization.

The process of recognition inside the university, as based on evidence provided by OOL providers, offers a variety of quality assurance scenarios already available from the good practices of most advanced universities in this area. Prior to the recognition, requirements for the process of open and online learning assessment, to ensure validity and recognition, include learner identification, learner authentication, process description, and the assessment process requirements. The collected evidence on how learning and assessment was done and what results were reached in many situations allows getting more objective data, if compared with traditional recognition process.

\section{Discussion and Conclusions}

Although different researchers (Camillieri \& Tannhäuser, 2012) and political documents (EU council recommendation, 2012; UNESCO Paris Declaration, 2012) stress the importance of creation of mechanisms for validation and recognition of OOL, our HE related research experts observe that only some universities are ready and have procedures to assess and recognize OOL. In general, OOL validation and recognition activities are more challenging than any accepted practices so far: they require human resources, digital tools, as well as closer cooperation with education stakeholders and quality assurance and standardisation of assessment on all levels.

Thus if a broader variety of OOL were to be recognized, major changes in internal university systems have to be introduced. Our research confirmed, however, that currently the OOL assessment and recognition at universities still depend on individual institutional policies and practices, as demonstrated by Conrad and McGreal (2012); and organized on individual basis that is not scalable, as indicated by Harris and Wihak (2018).

Elements leading to quality assurance in the process and result of assessment and recognition: identity verification of the learner; suitable supervised assessment; informative credentials; quality assurance; award of credit points; and Partnerships and collaboration with potentially recognizing institutions or bodies (Witthaus et al., 2016) were mentioned and discussed by our research experts. Some external trends, such as attitudes towards openness and trust in OOL, labour market flexibility and impact, and institutional readiness requirements: the readiness of institutional culture, systems and personnel (which were not mentioned in other theoretical sources) were also indicated. Our experts also emphasized that HE institutions are adapting too slowly to the changes in the labour market and learner and employer needs, and do not have trained HR capacity or will to recognize OOL.

Our research confirmed that the main aims of OOL are the same as the ones of open non-formal learning recognition, indicated by Witthaus et al. (2016): they are related to (i) entry to programmes offered by HEI; (ii) exemption from part of the programme or (iii) awarding a complete HE qualification. Our research experts also mentioned that it is considerably easier to get the recognition of OOL when aiming at free credits or entering 
programmes offered by HEI, than those which are a part of a minor or major programmes and lead to a qualification.

To conclude, research and practice of recognizing OOL in higher education are still emerging and are in a rather initial stage. Although OOL recognition procedures tend to be similar to traditional recognition, universities face some external challenges, e.g. those coming from the labour market, when enterprises tend to recognize the competencies of their employees and are more flexible compared to universities - universities are too slow to adapt to these changes in the market. The phenomenon of OOL lacks trust in the society and faces resistance from the universities. The lack of trust is the main obstacle of a successful recognition process, while traditional learning recognition practices are much more preferred and widely accepted.

The readiness of universities to recognize OOL means new structural changes, changes in institutional values and culture, change of attitude towards openness, transparency, sharing and use of learning/teaching content created by others, new modes of teaching and learning, curriculum restructuring, and strengthening networks with OOL providers. Information, data and qualitative evidence are the main requirements for OOL providers to ensure a successful process of OOL recognition in the university. These qualitative characteristics encompass the problem of OOL recognition and provide a response to the question we have formulated as the title of this paper.

For universities to stay competitive in the market our research findings suggest to be open, do research, and keep up to industry and society demands; participate in projects that allow innovation testing and application in the HE systems; analyse the existing systems and programmes and introduce data collection culture, issuing of digital badges or other digital credentials; and establish such systems for digital credentials that can be easily synchronized in the future; to think and work in collaboration with other universities and in networks.

\section{Acknowledgments}

The research has been implemented within the framework of the research project "Open and Online Learning for Digitalised and Networked Society" (project No. 09.3.3-LMT-K-712-01-0189) funded by the European Social Fund according to the activity "Improvement of researchers' qualification by implementing world-class R\&D projects' of Measure No. 09.3.3-LMT-K-712.

\section{References}

Bernard, H. R., \& Ryan, G. W. (2010). Analyzing qualitative data: Systematic approaches. SAGE Publications, Inc.

Camilleri, A. F., \& Tannhäuser, C. (2012). Assessment and recognition of open learning. In A. Meiszner, \& L. Squires (Eds.), Openness in Education (pp. 85-118). Emerald Group Publishing Limited. https://doi.org/10.1108/S2051-2295(2013)0000000004

CEDEFOP (2006). Validation and open educational resources (OER): Thematic report for the 2016 update of the European inventory on validation. https://doi.org/10.2801/80977

Chauhan, A. (2014). Massive open online courses (MOOCS): Emerging trends in assessment and accreditation. Digital Education Review, 25, 7-18. https://doi.org/10.5944/openpraxis.8.3.287

Cole, M. T., Shelley, D. J., \& Swartz, L. B. (2014). Online instruction, e-learning, and student satisfaction: A three year study. The International Review of Research in Open and Distributed Learning, 15(6). https://doi.org/10.19173/irrodl.v15i6.1748

Conrad, D., \& McGreal, R. (2012). Flexible paths to assessment for OER learners: A comparative study. Journal of Interactive Media in Education, 2012(2). https://doi.org/10.5334/2012-12

Council of the European Union. (2012). Council recommendation of 20 December 2012 on the validation of non-formal and informal learning. Official Journal of the European Union. Retrieved from https://eur-lex.europa.eu/legal-content/EN/TXT/?uri=CELEX\%3A32012H1222\%2801\%29

Creswell, J. W. (2013). Qualitative inquiry and research design: Choosing among five approaches. https://doi.org/10.1177\%2F1524839915580941

Friesen, N., \& Wihak, C. (2013). From OER to PLAR: Credentialing for Open Education. Open Praxis, 5(1), 49-58. https://doi.org/10.5944/openpraxis.5.1.22

Harris, J., \& Wihak, C. (2018). The recognition of non-formal education in higher education: Where are we now, and are we learning from experience? International Journal of e-Learning \& Distance Education, 33(1), 1-19. Retrieved from https://files.eric.ed.gov/fulltext/EJ1180057.pdf

Jupp, V. (2006). The SAGE dictionary of social research methods. https://doi.org/10.4135/9780857020116 
Kvale, S., \& Brinkmann, S. (2009). InterViews: Learning the craft of qualitative research interviewing (2nd ed.). Thousand Oaks, CA, US: Sage Publications, Inc.

Langen, F., \& Bosch, H. (2013). Massive open online courses: Disruptive innovations or disturbing inventions? Open Learning: The Journal of Open, Distance and e-Learning, 28(3). https://doi.org/10.1080/02680513.2013.870882

Mackintosh, W., McGreal, R., \& Taylor, J. (2011). Open Education Resources (OER) for assessment and credit for students' project: Towards a logic model and plan for action. In R. McGreal, W. Kinuthia, \& S. Marshall (Eds.), Open educational resources: Innovation, resource and practice (pp. 47-63). Retrieved from https://oerknowledgecloud.org/sites/oerknowledgecloud.org/files/pub_PS_OER-IRP_web.pdf

Radford, A. W., Robles, J., Cataylo, S., Horn L., Thornton, J., \& Whitfield, K. (2014). The employer potential of MOOCs: A mixed-methods study of human resource professionals' thinking on MOOCs. International Review of Research in Open and Distance Learning, 15(5), 1-25. https://doi.org/10.19173/irrodl.v15i5.1842

Silverman, D. (2007). Interpreting qualitative data (3rd ed.). SAGE Publications, Inc.

Simpson, O. (2013). Supporting students in online, open and distance learning. https://doi.org/10.4324/9780203417003

Tait, A. (2000). Planning student support for open and distance learning. Open Learning: The Journal of Open, Distance and e-Learning, 15(3), 287-299. https://doi.org/10.1080/713688410

Thorpe, M. (2002). Rethinking learner support: The challenge of collaborative online learning. Open Learning: The Journal of Open, Distance and e-Learning, 17(2), 105-119. https://doi.org/10.1080/02680510220146887a

UNESCO. (2012). UNESCO guidelines for the recognition, validation and accreditation of the outcomes of non-formal and informal learning. Hamburg: UIL. Retrieved from https://unesdoc.unesco.org/ark:/48223/pf0000216360

Witthaus, G., Inamorato dos Santos, A., Childs, M., Tannhauser, A-Ch., Conole, G., Nkuyubwatsi, B., \& Punie, Y. (2016). Validation of non-formal MOOC-based learning: an analysis of assessment and recognition practices in Europe (OpenCred).

\section{Copyrights}

Copyright for this article is retained by the author(s), with first publication rights granted to the journal.

This is an open-access article distributed under the terms and conditions of the Creative Commons Attribution license (http://creativecommons.org/licenses/by/4.0/). 